\title{
Simulation of flutter suppression for a transonic fan blade based on plasma excitation
}

\author{
Ying Liu, Xiaobo Zhang*, and Fei Zhang \\ Aeronautics Engineering College, Air Force Engineering University, Xi’an, 710038, China
}

\begin{abstract}
Along with the development of advanced high-performance aero-engines to the higher thrust-weight ratio, further improvement of stage load, the adoption of new materials and new lightweight structures, the aeroelasticity of blade structure is becoming more and more prominent. The high cycle fatigue failure of blades significantly reduces the structural reliability during the process of development and using. At the same time, a large number of failure forms of aero-engine experimental and server can be attributed to aeroelastic problems. Therefore, it is urgent to improve the aeroelastic stability of the blade. One of the most important factors is to suppress the airflow separation, but its mechanism is still unclear. Based on this, this paper combines the aerodynamic damping analysis of energy method with the plasma excitation simulation and references low-speed wind tunnel plasma expansion test to consider the effects of different exciter distributions and intensities on flutter. The results show that stall flutter is related to the flow separation, but the flow separation is not a key factor that determinates whether the flutters occurs or not. Flutter suppression is strongly correlated with the shock wave intensity, amplitude of first harmonic aerodynamic force, low-speed separation and aerodynamic work density. In addition, the relative distribution of the excitation field and the positive work zone also has a direct effect on the suppression of flutter.
\end{abstract}

Keywords: Stall flutter, Plasma excitation, Flutter suppression, Numerical simulation.

\section{Introduction}

Blade flutter is one of the fluid-induced aeroelastic self-excited phenomenon, which often occurs in the turbomachinery, especially for the transonic compressor blades. The main methods used to flutter analysis are empirical method, semi-empirical method, numerical prediction method, and test method, in which numerical method including the energy method, eigenvalue method and time domain method are commonly used in the engineering. Among them, the energy method has become the main tool for the blade flutter analysis in turbomachinery due to its clear physical concept, high accuracy and strong repeatability. The energy method was proposed by Carta ${ }^{[1]}$ and Bendikson ${ }^{[2]}$.

\footnotetext{
* Corresponding author: $\underline{x a z x b @ 163 . c o m}$
} 
Snyder ${ }^{[3]}$ used energy method to study the influence of Mach number, reduced frequency, installation angle and phase angle between blades on supersonic non-stall flutter and proved that the results obtained by the energy method are consistent with the experimental data. Zhang ${ }^{[4]}$ interpolated the structure obtained from the computational structure dynamics analysis on the fluid grid and applied the computational fluid dynamics (CFD) method to realize the unsteady analysis under the oscillating field, and the results show that the flutter boundary of the compressor blade predicted by the energy method agrees well with the test one.

The methods of compressor blade flutter suppression usually include mistuning the blade frequency, changing excitation conditions, improving blade vibration resistance, increasing structural damping and so on. For stall flutter, its flow field characteristics have a strong correlation with the unstable flow phenomena such as flow separation and leakage vortices. At the same time, the plasma flow control technology is a developing method on the compressor stall margin improvement. Experts and scholars think the plasma excitation has significant technological advantages such as short response time and short excitation frequency bandwidth. And it has broad application prospects in compressor stall margin improvement. Roy ${ }^{[5,6]}$ researched on the control model of atmospheric pressure surface plasma aerodynamic flow, the discharge dynamics of the plasma exciter and the courage to suppress the flow separation of plasma excitation. The research demonstrates of the influence of electricity generated by surface discharge on the flow of working gas, the decay process of a separation bubble was recorded for the first time. This work lays the foundation which the simulation method to the flow of common aerodynamic configurations. Corke ${ }^{[7-12]}$ through the basic experimental research on plasma flow control found that more than double the lift-drag ratio of the NACA 663-018 airfoil under the condition of Reynolds number of $7.9 \times 10^{4}$ had been given. Compering simulation test and actual, Liang et al. ${ }^{[13]}$ proved that the aerodynamic excitation is equivalent to the volume force with high accuracy which laid the foundation for the application of plasma flow control technology.

Therefore, this paper used fluid simulation software CFX to analyze the first stage rotor blades of the aero-engine compressor based on plasma aerodynamic excitation technology. Compressor characteristics has been obtained by the simulation to determine the typical stall flutter operating point for the later flutter flow field analysis. Through comparing the aerodynamic damping, aerodynamic work density distribution, static pressure distribution on the blade surface and aerodynamic separation of the flow field, the flutter characteristics of the compressor blades under the conditions with or without plasma excitation have been analyzed. And the mechanism of compressor stall margin improvement and flutter suppression have been explained.

\section{Basic theory}

\subsection{Energy method}

The basic idea of the energy method is assuming that the flutter occurs under a certain natural vibration mode of the rotor blade. The flutter is predicted by calculating the energy exchange between the blade and flow field in a vibration period. The blade vibration will divergence and the flutter will occur when the damping ratio is negative. Equation 1 gives the expression of aerodynamic work.

$$
W_{\text {aero }}=\int_{t_{0}}^{t_{0}+T} \int_{A} p \vec{v} \cdot \vec{n} d A d t
$$


where $P$ is the airflow pressure on the blade surface, $\vec{v}$ is the induced velocity caused by the vibration displacement imposed on the blade, $\vec{n}$ is the unit normal vector of the blade surface, $A$ is the blade surface area and $T$ is the vibration period. Equation 2 gives the calculation formula of aerodynamic modal damping ratio.

$$
\zeta_{\text {aero }}=-\frac{W_{\text {aero }}}{2 \pi q^{2} \omega^{2}}
$$

where $\zeta_{\text {aero }}$ is the aerodynamic modal damping ratio, $q$ is the regularized amplitude of the selected mode, $\omega$ is the natural circular frequency of the selected mode.

\subsection{Plasma excitation model}

This article used physiognomy model of Professor Soloviev ${ }^{[14]}$. Fig.1 shows the plasma exciter model. It should be noted that the SDBD (Surface Dielectric Barrier Discharge) model assume that it is uniform and constant along the $\mathrm{Z}$ coordinate axis. Equation 3 gives the calculation formula of the volume force.

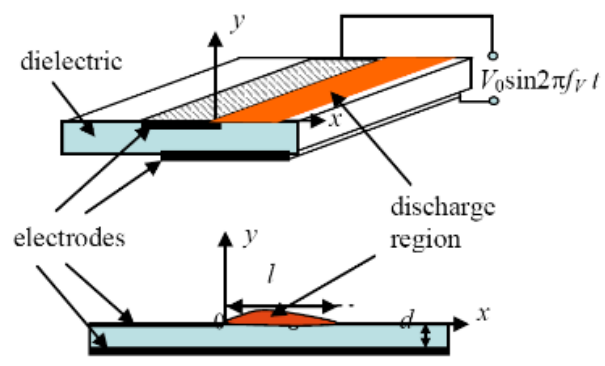

Fig. 1. SDBD model.

$$
\begin{aligned}
& f(x, y)=\theta(\bar{x}) \frac{F_{\varepsilon}}{x_{0} y_{0}} \overline{x y} e^{-(\bar{x}+\bar{y})}, \bar{x}=\frac{x}{x_{0}} ; \bar{y}=\frac{y}{y_{0}} \\
& F_{\varepsilon} \approx 4.75 \cdot 10^{-7} \frac{f_{v}[K H z]}{d[m m]}\left(V_{0}[k V]^{4}\right)\left(1-\frac{0.7}{V_{0}[k V]}\right)^{5}
\end{aligned}
$$

where $X_{0}, Y_{0}$ is the $X$ and $Y$ coordinates of the force max point, $\theta(\bar{x})$ is the heaviside function, $V_{0}$ is the voltage amplitude, $d$ is the dielectric thickness, $f_{v}$ is the voltage frequency.

\section{Results and analysis}

\subsection{Model}

A transonic compressor rotor blade model was used in this paper. The three-dimensional model of the whole flow channel is shown in Figure 2. The material property of the blade is as follows: the density is $7800 \mathrm{~kg} / \mathrm{m}^{3}$, the elastic modulus is $210 \mathrm{GPa}$ and the Poisson's ratio is 0.3 . 


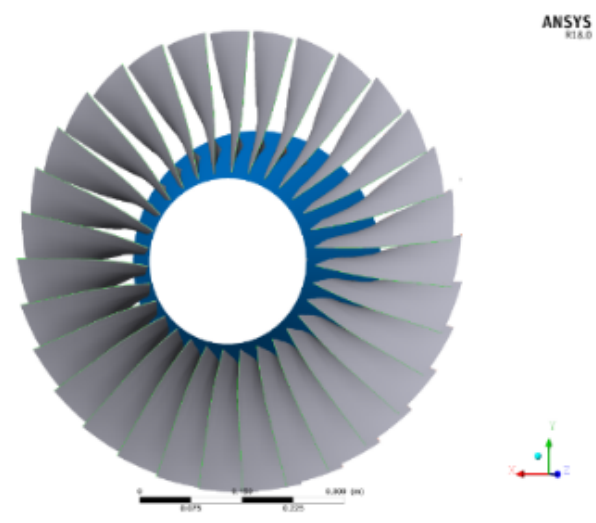

Fig. 2. Three-dimensional model of the full-channel blade.

\subsection{The Steady flow field analysis}

The design flow of the compressor is $65 \mathrm{~kg} / \mathrm{s}$. In the CFX calculation, the baffling effect of the compressor was simulated by the given outlet average static pressure. Therefore, the compressor approaches the choke boundary when the back pressure is reduced, and the compressor approaches the surge boundary when the back pressure is increased. The flow field was analysed by changing the back pressure in the outlet. The compressor characteristic curve is shown in Figure 3. The results show that the working-point of the maximum efficiency is the case of the back pressure under $104 \mathrm{kPa}$ and the working-points of stall flutter is the case of the back pressure under $113 \mathrm{kPa}$.
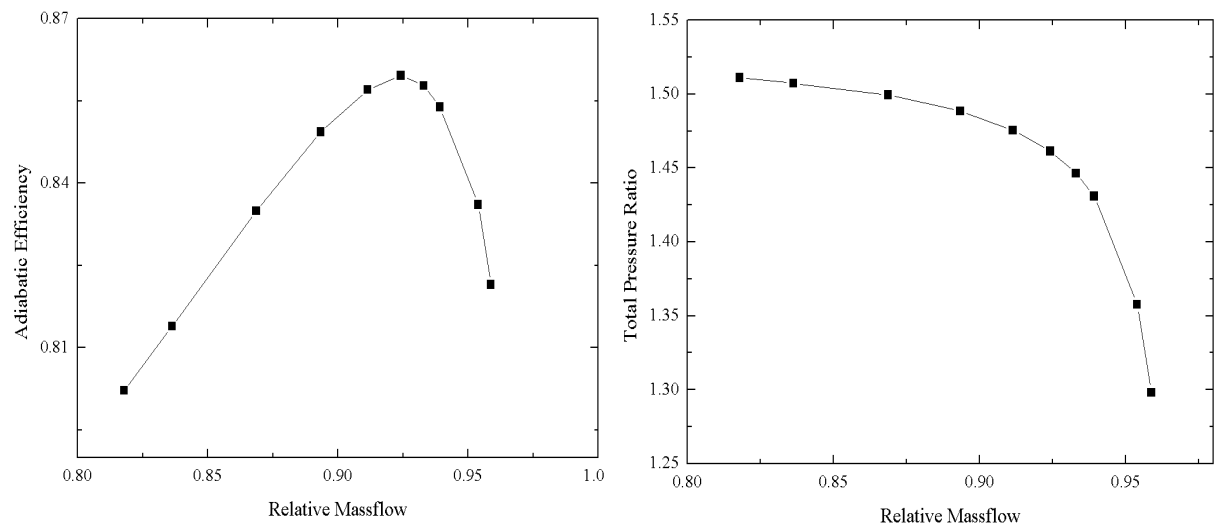

Fig. 3. Compressor characteristic curve.

This paper used typical stall flutter conditions that under the back pressure of $113 \mathrm{kPa}$ for analysis. Figure 4 shows the streamline and the vortex core which calculated based on the $\mathrm{Q}$ criterion. 


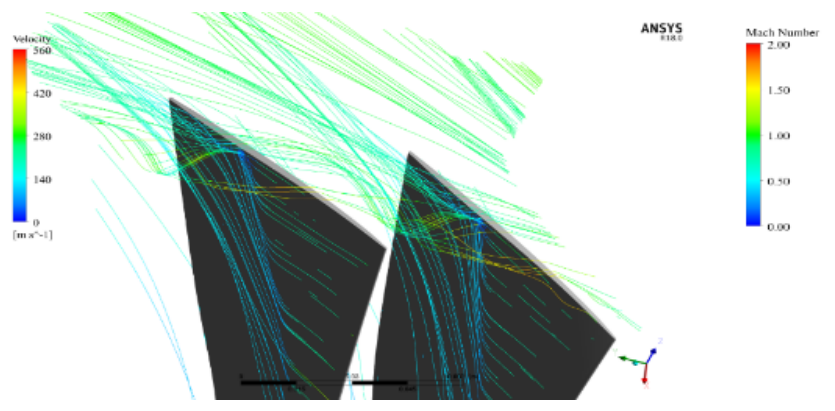

(a) The streamline of tip leakage flow

(b) Vortex core of tip leakage vortex

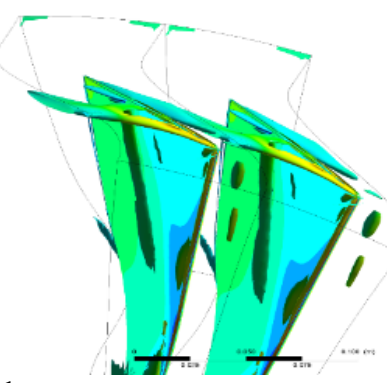

Fig. 4. Air flow in tip clearance.

Figure 5 shows the Mach number at 0.9 Span. Figure 6 shows the static pressure and streamline distribution on the blade surface. A comparison between Fig.5 and Fig. 6 can be found that the airflow separation after strong shock waves and a backflow area is generated at the tip of the blade. Combined with the known experiences, the backflow area will have a strong impact on flutter. Therefor, the following sections will focus on the tip clearance backflow area.

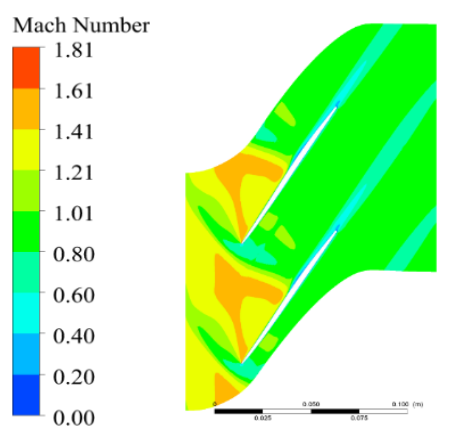

Fig. 5. Mach number at 0.9 Span

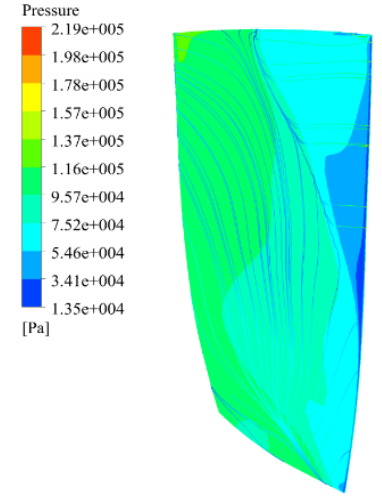

Fig. 6. Static pressure and streamline on blade surface

\subsection{The oscillation flow filed analysis}

This paper used the aerodynamic damping analysis method based on the energy method and take the seven harmonics into account to simulate the oscillation flow field. Figure 7 shows the $41^{\circ}$ ( 4 nodal diameters (ND), forward wave) phase of two blades. The result of the case under $113 \mathrm{kPa}$ back pressure in section 3.2 was used as the initial condition of the oscillating flow field. The first-order bending mode $(306 \mathrm{~Hz})$ and the maximum blade displacement $1 \mathrm{~mm}$ of the boundary condition under blade was given. The aerodynamic damping value of the flow field under different nodal diameters was obtained by the calculation formula of the damping ratio as shown in Figure 8. Fig. 8 shows the aerodynamic damping ratio under the interblade phase angle (IBPA) of $41^{\circ}$ (4ND, forward wave) is -0.0018 , which is the most prone case to flutter. The aerodynamic damping ratio of $-150^{\circ}$ (13ND, backword wave) IBPA is 0.0362 , which is the most stable operating case.

Figure 9 shows the static pressure distribution and the vortex core distribution on the suction surface of the blade in a period. For the convenience of observation, the isopressure line was given as a reference. By comparison, it can be seen that the shock wave and the tip clearance leakage vortex core moves to the leading edge in the first $1 / 2$ period. 
The shock wave and the tip clearance leakage vortex core moves to the trailing edge in the last $1 / 2$ period. It can be determined that the motion of vortex core is directly related to the position of shock wave and the airflow separation. Figure 10 shows the amplitude of the first harmonic aerodynamic force. Reslults show that the position of the shock wave in the oscillating flow field changes with the change of the flow field. Therefore, it can be seen that a local surface near the shock wave moves back and forth relative to the aerodynamic "strip" of the suction surface at different times which will result a large amplitude of aerodynamic fluctuations. The aerodynamic work density distribution in Figure 11. Compared with Fig 6, the distribution of aerodynamic force is basically the same as the shock wave. And the distribution of aerodynamic work density is basically the same as the first harmonic aerodynamic force which proved that the distribution of the first harmonic aerodynamic force and shock wave will both directly affect the aerodynamic work density.

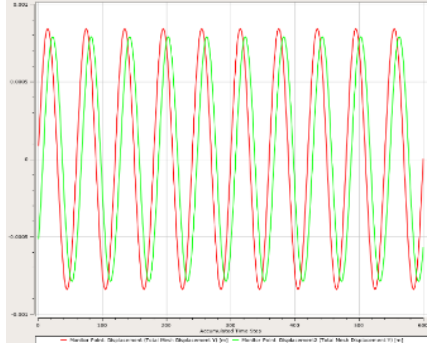

Fig. 7. Blade phase angle

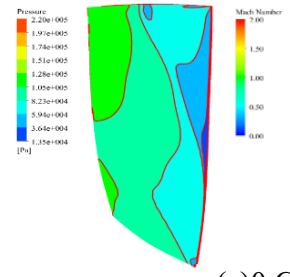

(a)0 Cycle
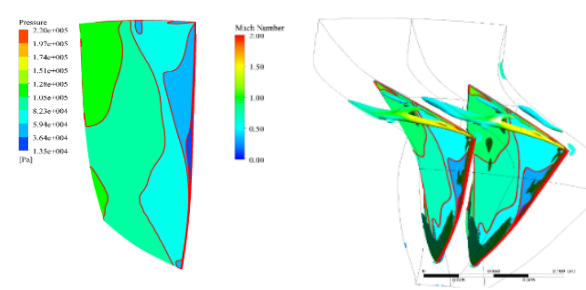

(c)1/3 Cycle
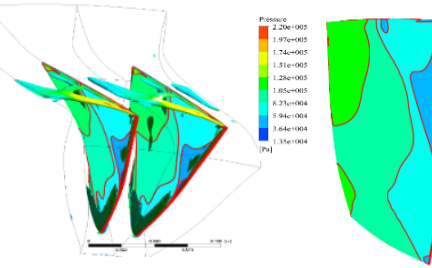

(b) $1 / 6$ Cycle
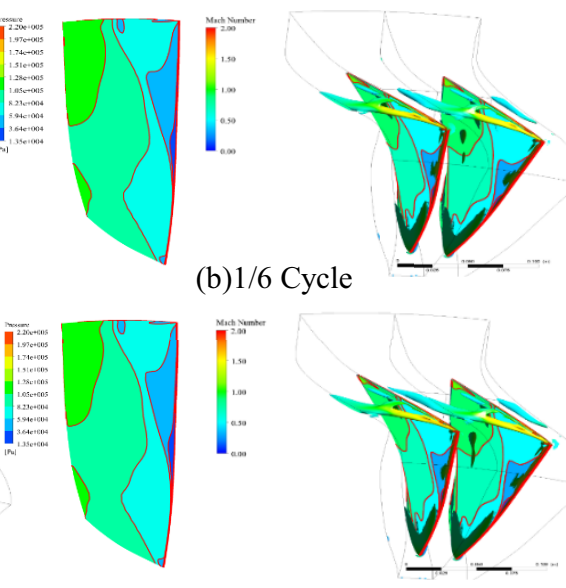

(d) $1 / 2$ Cycle
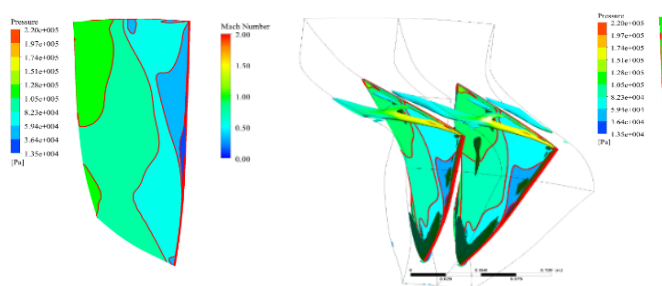

(e) $2 / 3$ Cycle
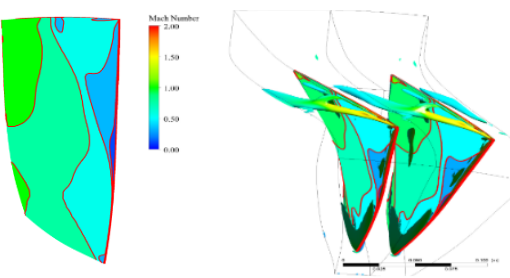

(f) $5 / 6$ Cycle

Fig. 9. Shock wave and vortex core motion in one cycle. 


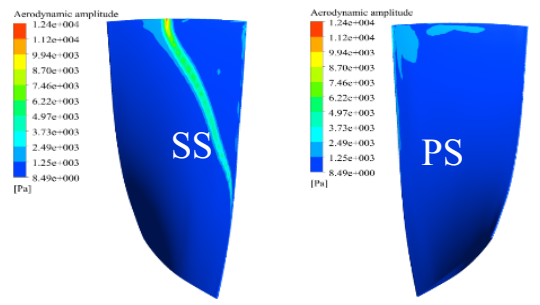

Fig. 10. First harmonic aerodynamic force.

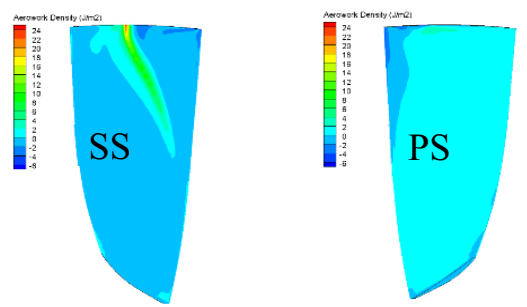

Fig. 11. Aerowork Density.

\subsection{Analysis of Flow Field Excited by Plasma}

Figure 12 shows the distribution of the excitation field. Based on the compressor stall margin improvement technology, the exciter was placed at the beginning of the flow separation, which may cause unsatisfactory results. Because after arranged the plasma exciter on the shroud, the airflow separation after the shock wave was obviously suppressed but the change of the aerodynamic damping value which with or without the excitation less than $3 / 1000$. Hence, the effect of the flutter suppression can be negligible.

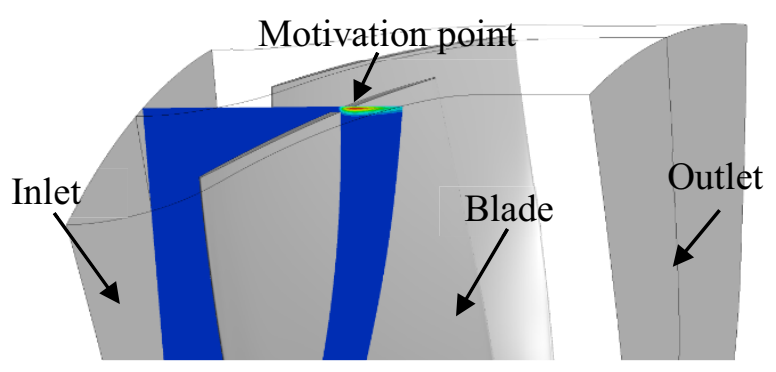

Fig. 12. Excitation field distribution.

Therefore, this article sets up the plasma exciter based on the aerodynamic work density distribution. Through a lot of practice, the author found that due to the existence of the strong shock waves, the plasma excitation needs to be controlled in the range of $15 \mathrm{~N} / \mathrm{M} \sim 17 \mathrm{~N} / \mathrm{M}$. The plasma exciters are divided into two parts, such as in front of the positive work zone and the back of the positive work zone. In order to reduce the effect of the shock wave, the exciter located in front of the positive work zone exerts a force on the leading edge to weaken the acceleration of the airflow and shock wave effect. In order to reduce low-speed recirculation zone, the exciter located behind the positive work zone exerts a force pointing the trailing edge to reduce the influence of low-speed region. Table 1 shows the results of the different excitation fields. Figure 13 shows the aerodynamic damping results under different excitation fields. The intensity of excitation under $15.28 \mathrm{~N} / \mathrm{M}$ was given in Case1-Case4.

The results show that Case1-Case 4 all have the effect on increasing the aerodynamic damping ratio and suppressing flutter. The positive work zone area is reduced under the four working conditions, but it can be found that the effect of Case 1 and Case4 are better than Case 2 and Case 3 because they are significantly reduced the positive work zone along the compressor radial and chord direction. Case 2 and Case 3 are mainly reduced positive work zone along the compressor chordwise significantly.

The results show that the size of the positive work area directly affects the aerodynamic damping ratio. Therefore, it can be considered that Case1 and Case4 have a better effect on flutter suppression under the same excitation intensity conditions. 
In order to study the incentive effects on the two distributions. Case 5 increase excitation intensity from 15 to $16.28(\mathrm{~N} / \mathrm{M})$ based on the distribution of Case4. Case 6 increase excitation intensity from 15 to 16.28 (N/M) based on the distribution of Case1. It can be seen that the flutter suppression effect of Case6 is better than Case5. The size of the positive work zone of Case 6 is smaller than Case 5 because of the 0.5 Span area excitation plays a key role in the reduction of the positive work area which is also the reason why Case 3 and Case2 have bad effects on the flutter suppression. In order to verify this point of view, Case 7 adds an excitor with a height of 0.5 blade on the basis of case 2 . This result is shown in Fig. 13 (g).

The results show that it is necessary for the excitation field to cover the entire positive work area and give exciters closer to the maximum value area, if the test want to get more effects of the flutter suppression. The main reason is that the closer to the maximum value area, the more obvious the effect of reducing the shock wave and suppressing the low-speed separation flow. The flutter suppression by plasma has two aspects: one is weakening the intensity of shock wave, the other is weaking the impact of the low-speed separation zone after the shock wave on the mainstream. It is clearly different from the mechanisms of the low-speed flow suppression. Therefore, it can be seen that stall flutter is related to flow separation, but flow separation is not a key factor in determining whether the blades flutter or not. Therefore, the excitation needs to cover the entire area of the region of the maximum value of the positive zone work zone. Only in this way, it can be possible to minimize the range of the positive work area to suppress the occurrence of flutter.

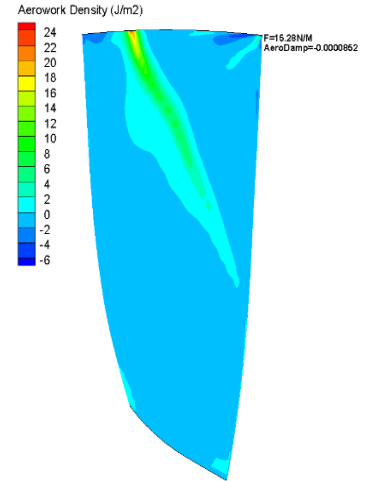

(a)Case1

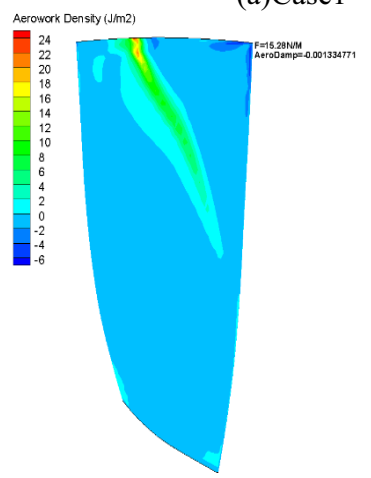

(c)Case 3
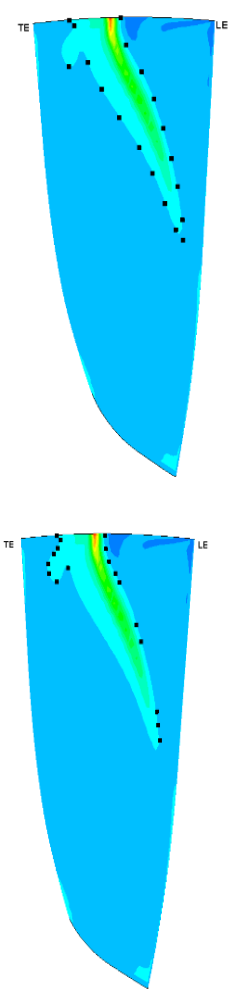

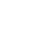
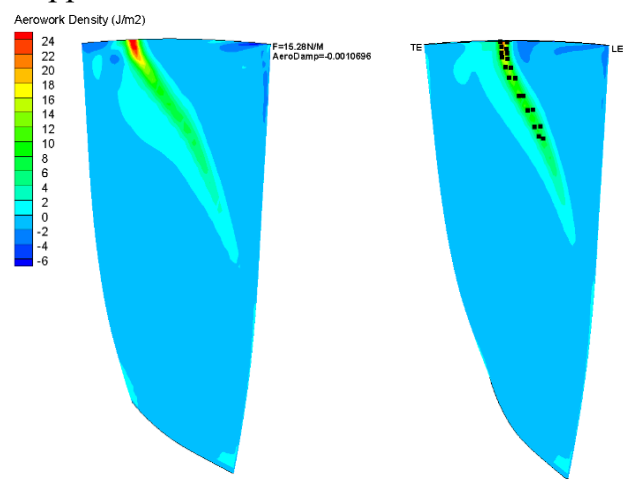

(b)Case2
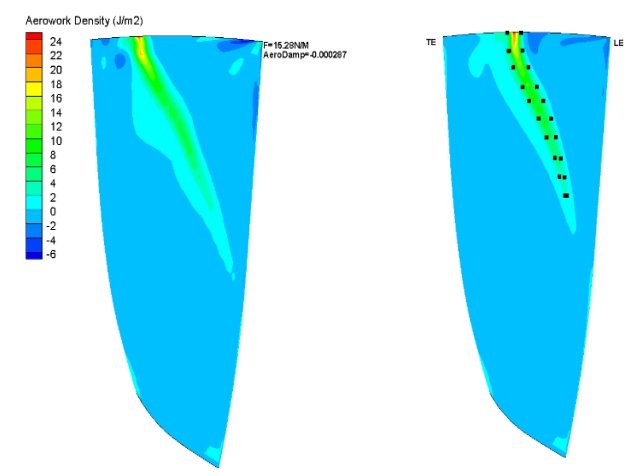

(d)Case4 


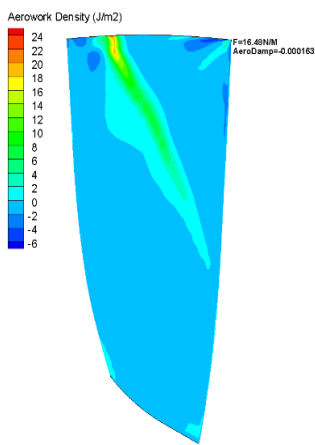

(e)Case5

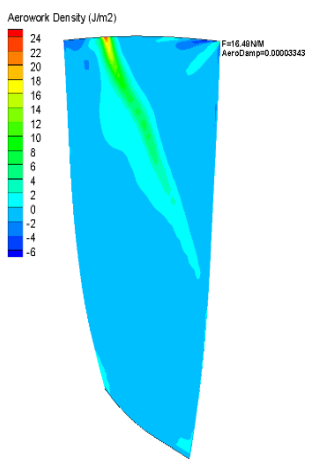

(f)Case6

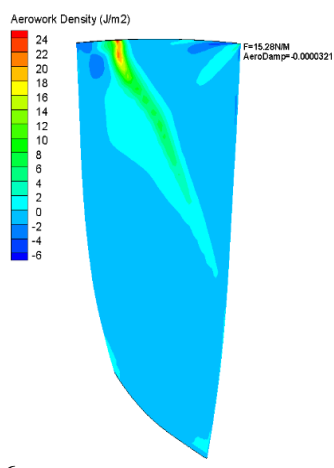

(g)Case7

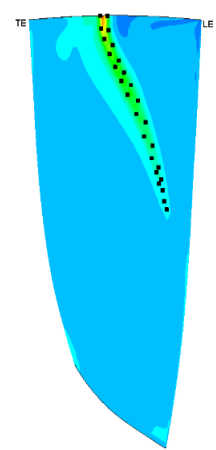

Fig. 13. Different excitation fields.

Table 1. Numerical value of excitation field and damping ratio for each case.

\begin{tabular}{cccccccc}
\hline & Case1 & Case2 & Case3 & Case4 & Case5 & Case6 & Case7 \\
\hline $\mathrm{F}(\mathrm{N} / \mathrm{M})$ & 15.28 & 15.28 & 15.28 & 15.28 & 16.48 & 16.48 & 15.28 \\
$\zeta_{\text {aero }}$ & $-0.85 \times$ & $-1.07_{\times 10^{-3}}$ & $-1.33_{\times 10^{-3}}$ & $-2.87 \times$ & $-1.6 \times$ & $3.34_{\times 10^{-5}}$ & $-3.2 \times 10^{-5}$ \\
\hline
\end{tabular}

\section{Conclusion}

In this paper, the aerodynamic damping analysis based on the traditional energy method and the numerical simulation analysis of plasma excitation has been conducted. The flutter suppression effect of plasma on transonic rotor blade was been investigated based on the known suppression effect of plasma on airflow separation under low-speed flow conditions. Compared with the typical stall flutter conditions of the steady and oscillating flow fields, the aerodynamic work density distribution and damping ratio of isolated rotor with or without plasma excitation was analysed. From the results, the conclusions can be concluded as follows:

(1) The results of steady-state flow field analysis show that leakage vortex exists in tip clearance. And its formation is directly related to the phenomenon of flow separation behind shock wave in the tip recirculation flow field

(2) The results of oscillating flow field show that the shock wave and the leakage vortex oscillate forward and backward along the chordwise in a period. It will lead to the relative position of blade and shock wave is different at each time. A local surface around the shock wave oscillate will induce a large amplitude of aerodynamic fluctuations. The occurrence of stall flutter can be attributed by the shock wave intensity, the distribution of aerodynamic work density and the amplitude of the first harmonic aerodynamic fluctuation.

(3) Considering a strong shock wave in the flow channel, the excitation intensity will be different from the value verified in the low-speed wind tunnel test. The excitation intensity of this paper is $15 \sim 17 \mathrm{~N} / \mathrm{M}$ which has a relatively obvious effect on the flutter suppression. The aerodynamic damping ratio can be improved for achieving the effect on the flutter suppression by reduce the intensity of the shock and suppressing the range of the low-speed zone. Finally, the results of numerical simulation indicate that in order to achieve a better effect on the flutter suppression, the excitation field needs to be covered near the whole area of the aerodynamic work in the region of the maximum value of the positive zone.

The work is supported by Natural Science Basic Research Plan in Shaanxi Province of China. (Program No.2020JQ-477) 


\section{References}

1. Carta F.O. Couple blade-disk-shroud flutter instabilities in turbojet engine rotors[J]. Journal of engineering for power, 1967, 89(03): 419-426.

2. Bendiksen O.O. Aeroelastic problems in turbomachines[C]. proceedings of the $31 \mathrm{st}$ structures, structural dynamics and materials conference, Long Beach, CA, USA, 1990.

3. L.E. Snyder, et al. Supersonic Unstalled Flutter In Fan Rotors; Analytical and Experimental Results[J]. Journal of Engineering for Gas Turbines and Power, 1974, 96(4):379-386. [4] Moffatt S, He L. On decoupled and fully-coupled methods for blade forced response prediction[J]. Journal of Fluids \& Structures, 2005, 20(2):217-234.

4. Zhang Xiaowei, Wang Yanrong, Xu Kening. Prerdiction method of blade flutter boundary in a compressor stage[J]. Journal of aerospace power, 2011, 26(02): 160-164.

5. Roy S, Gaitonde D V. Modeling surface discharge effects of atmospheric RF on gas flow control[R]. AIAA, 2006-0104.

6. Roy S, Gaitonde D. Multidimensional Collisional Dielectric Barrier Discharge for Flow Separation Control at Atmospheric Pressures[C]. Aiaa Fluid Dynamics Conference \& Exhibit, 2005.

7. Corke T, Matlis E. Phased plasma arrays for unsteady flow control[C]. Fluids Conference \& Exhibit, 2006.

8. Post M.L, Corke T.C. Separation Control on HIgh Angle of Attack Airfoil Using Plasma Actuators[J]. AIAA Journal, 2004.

9. Corke T C. Annual report of center for flow physics and control[J]. AIAA Journal, 2005.

10. Corke T C. Thomas F O. Enhanced design of turbo-jet LPT by separation control using phased plasma actuators[R]. NASA/CR-2003-212294.

11. Patel M.P, Sowle Z.H, Corke T.C, et al. Autonomous Sensing and Control of Wing Stall Using a Smart Plasma Slat[J]. Journal of Aircraft, 2007, 44(2):516-527.

12. Corke T, Mertz B, Patel M. Plasma Flow Control Optimized Airfoil[C]. 44th AIAA Aerospace Sciences Meeting and Exhibit. APS Division of Fluid Dynamics Meeting Abstracts, 2006.

13. Liang Hua, Li Yinghong, Wu Yun, et al. Numerical simulation of plasma aerodynamic actuation[J]. High voltage engineering, 2009, 35(05): 1071-1075.

14. V.R. Soloviev, V.M. Krivsov. Analytical and Numerical Estimation of the Body Force and Heat Sources Generated by the Surface Dielectric Barrier Discharge Powered by Alternating Voltage[C].6thEuropean conference for aeronatutics and space sciences, 2015. 